\title{
ESTUDO DA CORRELAÇÃO DOS INFECTADOS POR DENGUE EM 2019 NOS MUNICÍPIOS QUE COMPÕEM A GERÊNCIA REGIONAL DE SAÚDE DE LEOPOLDINA, MINAS GERAIS, NO CONTEXTO SOCIOECONÔMICO.
}

\author{
João Pedro Trajano Maciel - j.pedromec@hotmail.com \\ Lívia Iglésias Corrêa de Paiva - livia_icp@hotmail.com \\ Tiago Bittencourt Nazaré - tiago@unis.edu.br \\ Antônio Elízio de Oliveira - antonioelizio@terra.com.br
}

\section{RESUMO}

O presente artigo tem como finalidade realizar um estudo da correlação de dados referentes às taxas registradas de incidência da dengue nos municípios que fazem parte da Gerência Regional de Saúde de Leopoldina com variáveis socioeconômicas. Realizou-se a correlação de Pearson com as 232 variáveis contidas no Atlas do Desenvolvimento Humano no Brasil para identificar quais possuíam maior correlação com a Taxa de Incidência de dengue nos municípios analisados. No desenvolvimento dos mapas dos municípios, utilizou-se o softwareArcGis versão 10.8 e paraobter e estudar o nível de correlação (r) entre as variáveis, utilizou-se o software Minitab versão 19. Foram identificadas quatro variáveis relacionadas ao ensino de nível básico e uma ao esgotamento sanitário com maior correlação de Pearson dentre as demais. Para apresentação dos resultados, foi criadoum gráfico de Pareto com as 25 correlações mais fortes e identificou-se que as primeiras 10 variáveis representaram um total de $50 \%$ das causas do problema, porém, utilizaram-se as 5 primeiras para realização do artigo, que foram mostradas em uma tabela, com as respectivas correlações (r) e seu nível de significância (p).Pode-se concluir que se faz necessário que as Secretarias de Saúde dos municípios realizem mobilizações nas escolas de forma rotineira e forneçam materiais para que os professores possam abordar o tema com maior frequência com os alunos. Além disso, se mostrou necessário o acompanhamento de agentes de saúde em residências que se encontram em situação de precariedade no abastecimento de água e saneamento básico afim de orientar sobre os riscos e como se prevenir.

Palavras-Chave:Correlação de Pearson, Dengue, Gráfico de Pareto, Análise Exploratória, Variáveis socioeconômicas. 


\section{INTRODUÇÃO}

Segundo a Organização Pan-Americana da Saúde (2019), a dengue é uma infecção viral queprovoca doença febril aguda e pode até mesmo desenvolver complicações potencialmente letais. É transmitida pelos mosquitos fêmeas, principalmente da espécie Aedes aegypti e, em menor proporção, da espécie Aedes albopictus. A dengue é generalizada ao longo dos trópicos, porém os riscos locais se diferem por serem influenciados pela precipitação, temperatura e rápida urbanização não planejada.

São quatro diferentes sorotipos do vírus causador da dengue: DENV-1, DENV-2, DENV-3 eDENV-4. Quando o indivíduo acometido pela infecção se recupera, torna-se então imune aquele sorotipo adquirido. Contudo, após a recuperação, a imunidade cruzada para os outros sorotipos é somente parcial e temporária. Infecções posteriores aumentam o risco de que o indivíduo anteriormente acometido pelo vírus desenvolva dengue grave. (OPAS, 2019)

De acordo com o Boletim Epidemiológico de Monitoramento dos casos de dengue,Chikungunya e Zika de 08 de janeiro de 2020, publicado pela Secretaria de Estado de Saúde de Minas Gerais, em 2019 foram 3014 casos classificados como dengue com Sinais de Alarme e 276 casos foram classificados como Dengue Grave.

Este é um tema relevante para a sociedade pois o a dengue é uma doença endêmica presente emtodo território nacional e são inúmeros os casos da doença no Brasil. Pensando em fazer uma correlação entre a dengue e outras variáveis socioeconômicas, será realizada uma análise estatística, utilizando dados dos 15 municípios pertencentes a Gerência Regional de Saúde de Leopoldina, disponibilizados pela Secretaria de Estado de Saúde de Minas Gerais.

Os resultados obtidos através da mineração e análise estatística dos dados irão gerar informaçõesimportantes para saber qual sua relação com o contexto socioeconômico favorecendo a implementação de estratégias mais eficazes de controle do vetor por meio de ações promovidas pelas Secretarias Municipais de Saúde.

Os objetivos do presente artigo são: coletar o número de casos prováveis dedengue nos 15 municípios em estudo; calcular a taxa de incidência de dengue por município; obter dados socioeconômicos dos municípios; analisar o nível de correlaçãoentre as variáveis em estudo.

\section{REVISÃO DE LITERATURA}

\subsection{Atlas de Desenvolvimento Humano no Brasil}


Conforme o Programa das Nações Unidas para o Desenvolvimento (2020), o Atlas de Desenvolvimento Humano no Brasil é uma plataforma de consulta de mais de 200 variáveis socioeconômicas, incluindo o Índice de Desenvolvimento Humano Municipal para 5.565 municípios brasileiros, além de consolidar os indicadores para as Unidades da Federação e regiões metropolitanas. Foi desenvolvido pela parceria entre o Programa das Nações Unidas para o Desenvolvimento (PNUD), o Instituto de Pesquisa Econômica Aplicada (Ipea) e a Fundação João Pinheiro

“O Atlas traz os dados dos Censos Demográficos de 1991, 2000 e 2010, oferecendo um panorama da conjuntura socioeconômica do país nas últimas duas décadas, promovendo o debate sobre desenvolvimento e apoiando o planejamento da gestão pública." (ATLAS DO DESENVOLVIMENTO HUMANO NO BRASIL, 2013)

\subsection{Trabalhos relacionados}

Existem hoje diversos artigos que analisam o desenvolvimento da dengue em várias regiões do país. A respeito do estudo sobre retrospectiva histórica de dengue no Brasil, Câmara et al. (2007) fez uso do software de análise estatística Minitab 14 afim de gerar informações gráficas e estudos estatísticos, como distribuição normal e ANOVA,analisando características regionais e dinâmicas. O autor, através de suas análises identificou uma expressiva taxa de ocorrência sazonal durante os meses mais quentes em todas as regiões do país, efeito correlacionado com o ciclo de reprodução do mosquito transmissor, porém, apenas na região Nordeste obteve-se maior número de notificações no segundo trimestre. A partir do segundo semestre, Câmara et al. (2007) verificou que o número de notificações de casos reduziusubstancialmente.

Em outro artigo, relacionado a dengue com o contextosocioeconômico no município do Rio de Janeiro, foi calculada a taxa média de incidência de dengue entre as semanas epidemiológicas $48^{\mathrm{a}}$ de 2001 e $20^{\mathrm{a}}$ de 2002 e posteriormente foi realizada uma correlação de Pearson entre essa taxa média encontrada e as variáveis socioeconômicas. Após isso, foi feita uma análise estatística de regressão linear múltipla e espacial a fim de apontar quais regiões e quais variáveis possuíam alguma correlação direta. De acordo com Almeida, Medronho e Valencia (2009), como resultado de seus estudos, identificou que os bairros da zona oeste do município apresentaram maior correlação com a doença, sendo a única variável com correlação significativa o percentual de domicílios ligados à rede sanitária de esgoto. 
Brassolati e Andrade (2002) em seu estudo fazem uma abordagem educativa em relação a prevenção da dengue em Campinas. Foi realizado um curso de 20 horas com os professores para que estes pudessem trabalhar o assunto com seus alunos e estes com seus familiares. Neste curso foi fornecido material didático e armadilhas para vigilância. Como resultado, foi avaliada em até 1 ano após o curso a redução e até mesmo eliminação de locais de criação dos vetores da doença em ambientes escolares e também para uma amostra das residências dos estudantes.

Silva e Andrade (2014) realizaram a coleta de dados através das fichas de notificação e investigação de dengue para os casos no município de Paripiranga/BA e através dessa pesquisa descritiva individual, identificaram que a maior parte da população acometida pela dengue dispunha do ensino fundamental a médio completo, os autores também notaram que a dengue apresentava um padrão sazonal, onde o primeiro trimestre do ano apresentou maior número de casos.

Pereira Júnior, Oliveira e Maia (2017), através do trabalho buscam identificar fatores que possam influenciar na dinâmica da dengue e da malária entre os frequentadores da Praça São Francisco em Marabá, Pará além de quantificar os casos das doenças neste local.Os dados foram coletados através de 400 formulários preenchidos no local de estudo e realizaram um levantamento bibliográfico.Com a análise dos dados coletados obtiveram-se os seguintes fatores influenciadores das doenças: precipitação, saneamento, falta de visitação dos agentes comunitários de saúde e falta de envolvimento da população. Como resultado, a pesquisa apontou que o saneamento básico associado ao não tratamento de água e esgoto e o acúmulo de resíduos sólidos é o principal fator relacionado a dinâmica da dengue e malária no município.

Na tese de Rocha (2011) foi realizada uma descrição da incidência de dengue e da infestação predial pelo mosquito Aedes aegypti entre os anos de 2000 a 2007, através de dados secundários, em relação as variáveis sexo, faixa etária e escolaridade. O coeficiente de incidência e o índice de infestação predial foram correlacionados com variáveis climáticas, demográficas, ambientais e socioeconômicas. Além disso também aplicou técnicas de geoprocessamento para avaliar a autocorrelação espacial da incidência de dengue na cidade. Como resultado obteve que há uma elevada incidência de dengue e infestação predial pelo Aedes aegypti, que o sexo dos afetados não influencia na incidência de dengue e a faixa etária mais acometida são entre 15 e 49 anos e alunos que não concluíram o ensino médio. Notou-se que há um padrão sazonal com elevação do índice em períodos de chuva e regressão nos 
períodos mais secos. $\mathrm{O}$ aumento nos casos foi significante em áreas em que há baixo índice de abastecimento de água.

\section{DADOS PARA ESTUDO}

O local de estudo do presente artigo engloba quinze municípios da Zona da Mata em Minas Gerais, que compõem a Gerência Regional de Saúde de Leopoldina. São eles: Além Paraíba, Argirita, Astolfo Dutra, Cataguases, Dona Euzébia, Estrela Dalva, Itamarati de Minas, Laranjal, Leopoldina, Palma, Pirapetinga, Recreio, Santo Antônio do Aventureiro, Volta Grande e Santana de Cataguases. Estes municípios possuem uma área total de 4.074,932 $\mathrm{km}^{2}$ de acordo com o Instituto Brasileiro de Geografia e Estatística (IBGE, 2019).

\section{MATERIAIS E MÉTODOS}

\subsection{Materiais}

Os casos confirmados de dengue utilizados no estudo foram originados dos arquivos da Secretaria de Estado de Saúde de Minas Gerais referente a atualização de dados do dia 14 de janeiro de 2020. É importante pontuar que se tratam de dados dinâmicos, sujeitos a alterações.

Os dados socioeconômicos coletados de cada município correspondem ao Censo de 2010 foram retirados do Atlas do Desenvolvimento Humano no Brasil.

Para obtençãodos mapas dos 15 municípios estudados foi utilizado o software ArcGis 10.8, que é um software que permite trabalhar com mapas e informações geográficas.

Para calcular e analisar o nível de associação entre as variáveis, utilizou-se o software Minitab 19.

Foi utilizado o Gráfico de Pareto para apresentação dos resultados obtidos com a correlação de Pearson. Segundo Lins (1993), o Gráfico de Pareto é um gráfico de barras que coloca cada causa, que contribui para um problema, em ordem crescente de ocorrência ou influência.

\subsection{Métodos}


No software ArcGis 10.8 foi necessário utilizar o arquivo shapefile de todos os municípios do estado de Minas Gerais, selecionar e recortar os municípios de interesse e em seguida, através da ferramenta Merge, realizar a união dos mesmos (Figura 1).

Figura 1. Taxa de incidência de dengue em 2019 por municípios que compõem a Gerência
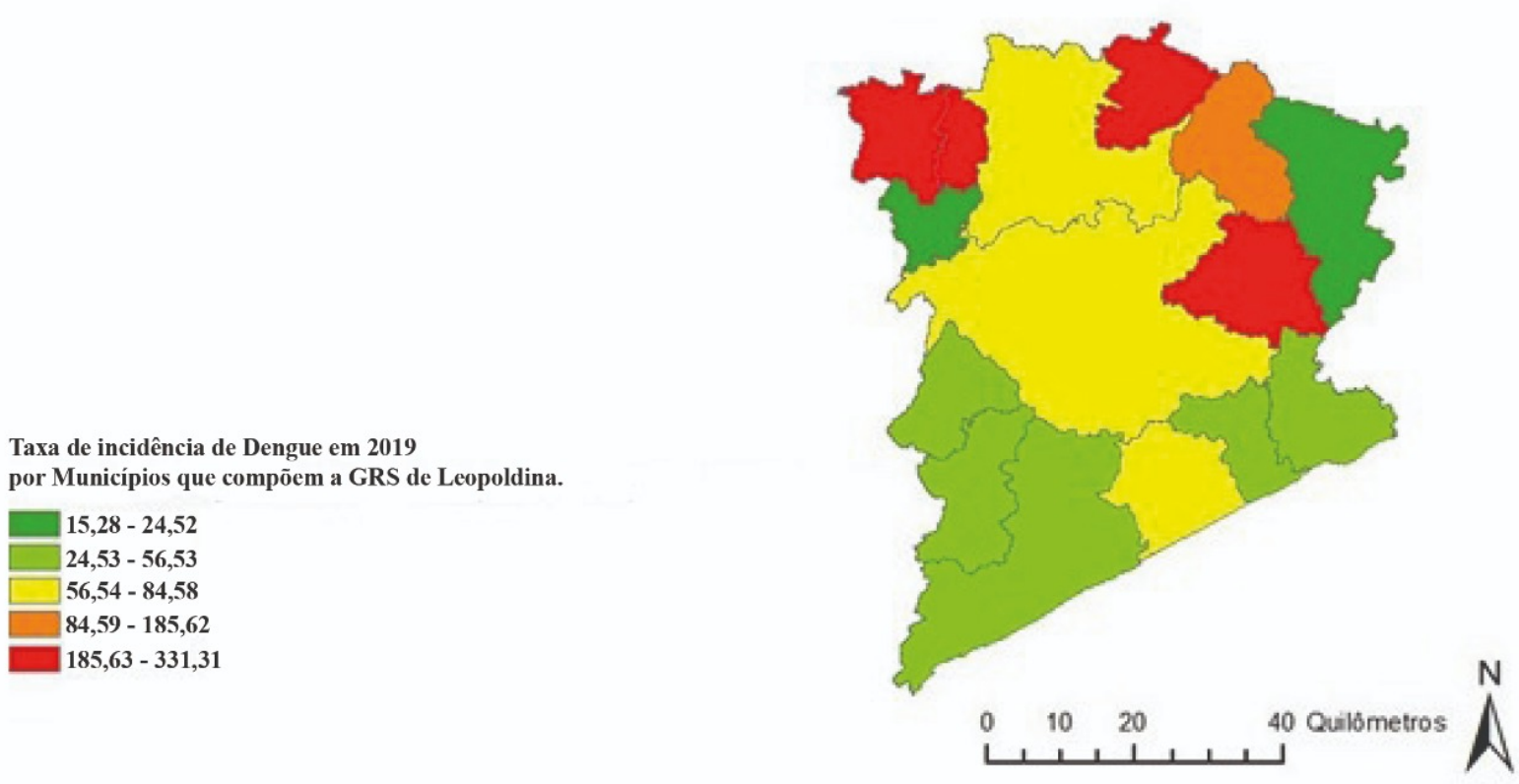

Regional de Saúde de Leopoldina

Fonte: Próprios autores (2020)

O primeiro passo para a criação do estudo foi analisar todas as áreas de cobertura de cada município que compõe a Gerência Regional de Saúde de Leopoldina e a distribuição populacional para a região em questão. Para isso, os mapas foram obtidos de acordo com o último relatório do censo gerado (2010), por meio do acervo proporcionado pelo IBGE Geociências (IBGE, 2010). O mesmo é dividido por diversos setores censitários e cada setor possui seu arquivo no formato shapefile.

Para haver um maior entendimento do padrão de distribuição da dengue na área geográfica em estudo, realizou-se a correlação de Pearson entre as 232 variáveis socioeconômicas e a taxa de incidência de dengue de cada município, cujo índice de correlação adotado foi de $\alpha=0,005$.

“O coeficiente de correlação Pearson (r) varia de -1 a 1. O sinal indica direção positiva ou negativa do relacionamento e o valor sugere a força da relação entre as variáveis." (FIGUEIREDO FILHO, D. B.; SILVA JUNIOR, J.A.,2010). Para Dancey e Reidy (2006), 
quando o valor de $\mathrm{r}$ está entre $\mathrm{r}=0,1$ e 0,3 , pode-se considerar a correlação fraca, $\mathrm{r}=0,4$ até 0,6 a correlação é moderada, $r=0,7$ forte e $r=1$ muito forte.

Em seguida, foi gerado o Gráfico de Pareto utilizando o MS-Excel 2016com as 25 variáveis que apresentaram maior correlação com a doença. Posteriormente, as cinco variáveis que tiveram maior correlação entre as 25 foram escolhidas para discussão do trabalho.

\section{RESULTADOS E DISCUSSÕES}

O Gráfico de Pareto (Figura 2) gerado mostra o nível de correlação das variáveis com a taxa de incidência de dengue. Analisando o gráfico, pode-se notar que as primeiras 10 variáveis representam um total de $50 \%$ das causas do problema, porém para o estudo utilizouse as primeiras 5 variáveis que apresentaram maior correlação de Pearson.

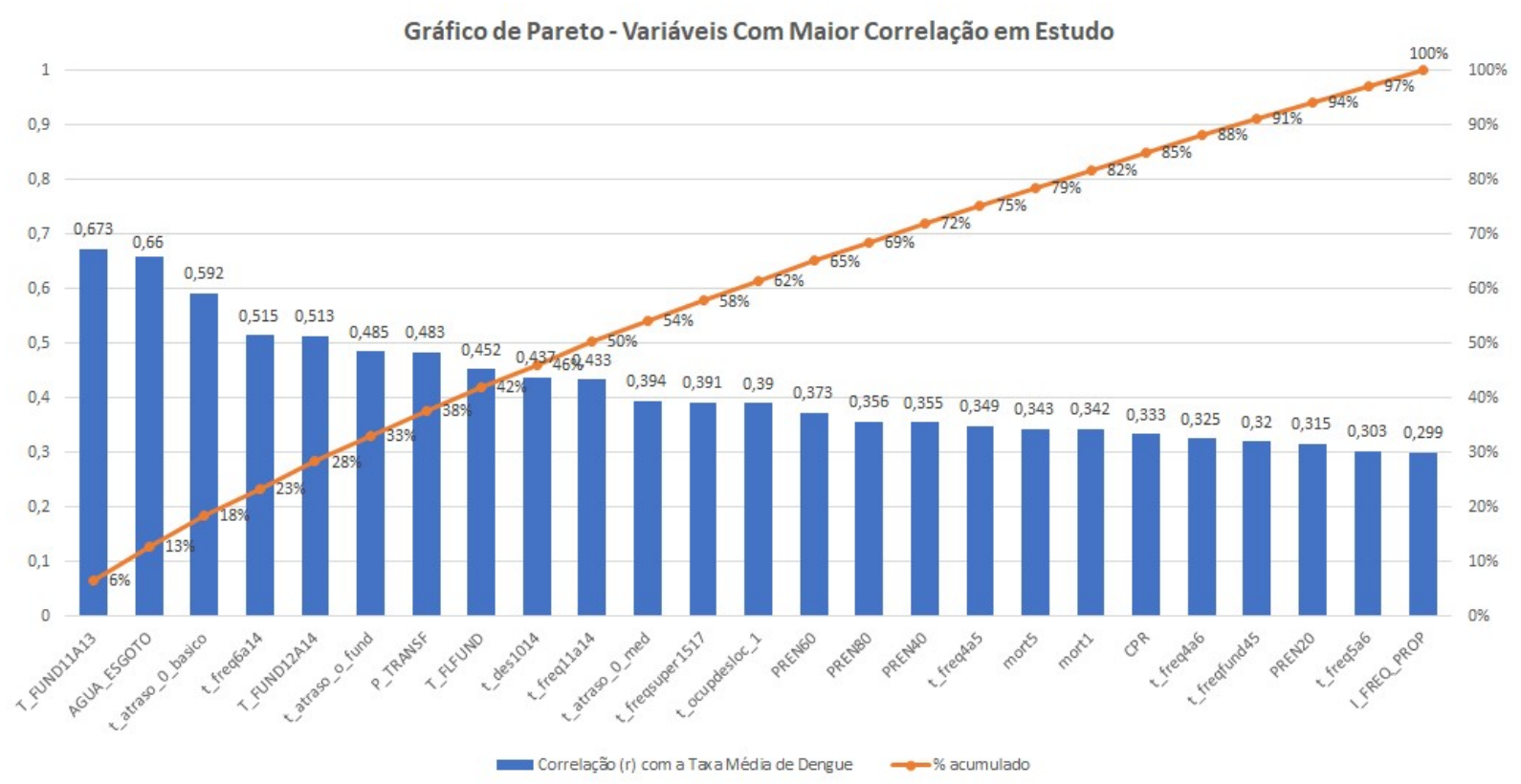

Figura 2. Gráfico de Pareto

Fonte: Próprios autores (2020)

Ao encontrar o coeficiente de correlação de Pearson (r) e o nível de significância (valor p) das 5 variáveis em estudo com a taxa de incidência de dengue dos municípios da GRS - Leopoldina (Tabela 2) pode-se observar que houve uma correlação positiva entre a taxa de incidência da doença e as demais variáveis, já que os valores encontrados foram todos variando de 0 a 1 . Em relação a força de correlação destas variáveis selecionadas pode-se notar que a correlação é moderada, considerando a convenção estabelecida por Dancey e Reidy (2006). 
Tabela 1. Coeficiente de correlação de Pearson (r) e nível de significância (valor p)

\begin{tabular}{|c|c|c|c|c|c|}
\hline \multirow{3}{*}{ Municípios da GRS - Leopoldina } & T_FUND11A13 & T_FUND12A14 & t_atraso_0_basico & t_freq6a14 & AGUA_ESGOTO \\
\cline { 2 - 7 } & 0,673 & 0,513 & 0,592 & 0,515 & 0,66 \\
\cline { 2 - 7 } & 0,006 & 0,05 & 0,02 & 0,05 & 0,007 \\
\hline
\end{tabular}

Fonte: IBGE (2010)

As 5 variáveis selecionadas, que apresentaram maior correlação com a dengue, foram as seguintes: Percentual da população de 12 a 14 anos de idade frequentando os anos finais do fundamental ou que já concluiu o fundamental (T_FUND12A14); Percentual da população de 11 a 13 anos de idade frequentando os anos finais do fundamental ou que já concluiu o fundamental (T_FUND11A13); Taxa de atendimento escolar da população de 6 a 14 anos de idade (T_FREQ6A14); Percentual da população de 6 a 17 anos de idade frequentando o ensino básico que não tem atraso idade-série (T_ATRASO_0_BASICO); Percentual de pessoas em domicílios com abastecimento de água e esgotamento sanitário inadequados (AGUA_ESGOTO).

Através dos resultados da correlação obtidos pode-se perceber que a dengue afeta adolescentes de 11 a 14 anos que estão cursando o ensino fundamental e também crianças a partir de 6 anos a adolescentes de 14 anos que frequentam escola. Brassolati e Andrade (2002) em seu estudo observaram que $61,5 \%$ dos professores do ensino fundamental de Campinas entrevistados não haviam trabalhado o assunto sobre a dengue com seus alunos devido à "falta de material informativo". Para Rocha (2011), é necessário entender a que condições ambientais e socioeconômicas os grupos de escolaridade pertencem, isso proporcionaria um entendimento mais detalhado de quais causas poderiam estar atreladas à transmissão da doença.

Por meio da correlação de Pearson também se verificou uma correlação da dengue com a população de 6 a 17 anos de idade que frequenta o ensino básico que não tem atraso idade-série. Este dado nos mostra que, não necessariamente o indivíduo precisa ter repetido algum ano escolar para se encaixar no padrão citado anteriormente, em que estudantes do ensino fundamental são acometidos por esse vírus, além deles também se encaixam nessa variável os alunos do Ensino Médio. Silva e Andrade (2014), em seu trabalho, após analisarem 512 fichas de notificação e investigação de dengue no período de janeiro de 2008 a abril de 2012 no município de Paripiranga, Bahia, verificaram que a maioria da população acometida pela dengue possuía ensino fundamental a médio completo e foram infectados no 
primeiro semestre do ano, que corresponde ao período de maior pluviosidade no município. Este resultado assemelha-se aos resultados do trabalho de Câmara et al. (2007), presente nos trabalhos relacionados, que verificou que o número de notificações de casos reduziu substancialmente no segundo semestre do ano estudado.

Também se notou uma correlação média positiva com a porcentagem das pessoas que não possuíam abastecimento de água e esgotamento sanitário adequados em seus domicílios, esse resultado se confirma com demais estudos realizados no Brasil, como por exemplo, Pereira Júnior, Oliveira e Maia (2017) em seu artigo identificou que no distrito de Cidade Nova, pertencente ao município de Marabá - PA que fatores ambientais como saneamento básico estava interligado com a dengue, segundo o autor, tal fator influencia na concentração de resíduos muitas vezes sólidos.

Garrafas, copos, pneus, comumente encontrados jogados em lixões, córregos e rios, servem de local para acúmulo de água, consequentemente, servindo de criadouro para o mosquito. A falta de fornecimento contínuo de água faz com que a população necessite estocar volumes suplementares em caixas d'água, tonéis e poços, esses reservatórios de água parada são conhecidos por serem locais apropriados para sua procriação.

Somado a esses fatores, Câmara et. al. (2007) identificou que em todas as regiões do país, exceto Nordeste, a taxa de ocorrência sazonal ocorre durante os meses mais quentes do ano, respectivamente, primeiro trimestre do ano. Os três primeiros meses do ano também estão associados a época de chuva nessas regiões, onde a taxa de reprodução do vetor transmissor se eleva consideravelmente. Esse fator climático (chuva) associado ao ambiental (saneamento básico) podem agravar os casos da dengue.

\section{CONCLUSÃO}

O desenvolvimento do presente estudo possibilitou a descoberta de possíveis causas para a disseminação do Aedes eegypti, através da correlação de Pearson realizada entre a taxa de incidência da dengue e variáveis socioeconômicas referente a 15 municípios mineiros no ano de 2019. Sabe-se que a dengue é uma infecção viral que pode até mesmo desenvolver complicações letais, além de estar presente em todo o território nacional, portanto é de suma importância que estudos relacionados a este tema estejam sempre em discussão na sociedade.

Por meio da análise das correlações, obtiveram-se cinco variáveis que apresentaram maior correlação com taxa de incidência de dengue. Pode-se notar que quatro destas estão relacionadas ao ensino de nível básico e uma em relação a abastecimento de água e 
esgotamento sanitário inadequados. Com isso, realizou-se uma pesquisa bibliográfica em outros estudos que identificaram através de suas análises e resultados, correlações semelhantes às identificadas no atual artigo para que se pudesse levantar possíveis soluções afim de mitigar os casos.

Em relação as variáveis pertinentes ao nível de ensino dos infectados pela dengue, pode-se atribuir ao fato de que os alunos que frequentam o nível básico de ensino (fundamental e médio) não estariam sendo devidamente orientados sobre formas de prevenção à doença até mesmo devido à falta de materiais informativos a respeito do assunto.

Sobre o abastecimento de água e esgotamento sanitário inadequados estarem ligados aos casos de dengue, atribui-se ao fato de que indivíduos que não tem acesso a água tratada e encanada estoquem água como em tonéis ou poços para ser usada posteriormente e as residências que não possuem esgoto são mais suscetíveis ao acúmulo de resíduos sólidos em seu quintal como garrafas, copos, pneus. Tais atitudes fazem com que aumente a propagação de lavas do mosquito Aedes aegypti e a doença dissemine.

Os objetivos do trabalho foram alcançados, pois, foi possível correlacionar estatisticamente a taxa de incidência de dengue em 2019 nos municípios pertencentes a Gerência Regional de Saúde de Leopoldina com variáveis de ordem socioeconômica através da coleta do número de casos prováveis de dengue, do cálculo da taxa de incidência de dengue, da obtenção dos dados socioeconômicos dos municípios e da análise do nível de correlação entre as variáveis em estudo.

Através dos resultados obtidos, fica evidente que a educação associada ao combate à dengue deve ser executada de maneira participativa e constante, mesmo fora dos períodos epidêmicos, demostrando a necessidade de cooperação entre os serviços de saúde, alunos e professores. Além disso, os agentes comunitários de saúde e de controle de endemias mostram-se importantes aliados para o combate da dengue através de uma fiscalização rotineira somado à uma conscientização domiciliar. 


\section{REFERÊNCIAS BIBLIOGRÁFICAS}

ALMEIDA, A. S.; MEDRONHO, R. A.; VALENCIA, L. I. O. Análise espacial da dengue e o contexto socioeconômico no município do Rio de Janeiro, RJ. Rev. Saúde Pública, São Paulo, v. 43, n. 4, p. 666-673, Ago. 2009. Disponível em: $<$ http://www.scielo.br/scielo.php?script=sci_arttext\&pid=S0034$89102009000400013 \& \operatorname{lng}=\mathrm{en} \& n r m=$ iso $>$. Acesso em: 21 abr. 2020.

ATLAS DO DESENVOLVIMENTO HUMANO NO BRASIL. O Atlas. 2013. Disponível em:<http://atlasbrasil.org.br/2013/pt/o_atlas/o_atlas_/>. Acesso em: 03 jul. 2020.

BRASSOLATTI, R.C.; ANDRADE, C. F. S. Avaliação de uma intervenção educativa na prevenção da dengue. Ciênc. saúde coletiva, São Paulo, v. 7, n. 2, p. 243-251, 2002 . Disponível em:

$<$ http://www.scielo.br/scielo.php?script=sci_arttext\&pid=S1413-81232002000200005\&lng=en\&nrm=iso $>$. Acesso em: 29 set. 2020.

CAMARA, F. P.; THEOPHILO, R.L.G.; SANTOS, G.T.; PEREIRA, S.R.F.G.; CAMARA, D.C.P.; MATOS, R.R.C. Estudo retrospectivo (histórico) da dengue no Brasil: características regionais e dinâmicas. Rev. Soc. Bras. Med. Trop., Uberaba, v. 40, n. 2, p. 192-196, Abr. 2007. Disponível em: $<$ http://www.scielo.br/scielo.php?script=sci_arttext\&pid=S0037-86822007000200009\&lng=en\&nrm=iso $>$. Acesso em: 26 abr. 2020.

DANCEY, C. P.; REIDY, J. Estatística sem matemática para psicologia. Porto Alegre: Artemed, p. 178-216, 2006.

FIGUEIREDO FILHO, D. B.; SILVA JUNIOR, J.A. Desvendando os Mistérios do Coeficiente de Correlação de Pearson (r). Revista Política Hoje, [S.1.], v. 18, n. 1, jan. 2010. ISSN 0104-7094. Disponível em: $<$ https://periodicos.ufpe.br/revistas/politicahoje/article/view/3852>. Acesso em: 02 jul. 2020.

IBGE - Instituto Brasileiro de Geografia e Estatística.Cidades e Estados. 2019.Disponível em: $<$ https://www.ibge.gov.br/cidades-e-estados/>. Acesso em: 26 abr. 2020.

IBGE - Instituto Brasileiro de Geografia e Estatística. Geociências. 2010. Disponível em: $<$ https://www.ibge.gov.br/geociencias/downloads-geociencias.html>. Acesso em: 26 abr. 2020.

LINS, B. F. E. Ferramentas básicas da qualidade. Ciência da Informação, v. 22, n. 2, 1993.

OPAS - Organização Pan-Americana da Saúde. Folha informativa - Dengue e dengue grave. Brasília: OPAS/OMS, 2019. Disponível

em: $<$ https://www.paho.org/bra/index.php?option=com_content\&view=article\&id=5963:folha-informativadengue-e-dengue-grave\&Itemid=812>. Acesso em: 05 abr. 2020.

PEREIRA JÚNIOR, A. P.; OLIVEIRA, G. P.; MAIA, J. O. Fatores ambientais (lixo), climáticos (chuva) e a evolução da dengue e malária: o caso da praça são francisco, Cidade Nova, Marabá-PA. Enciclopédia Biosfera, v. 14, n. 25, 2017. Disponível em:

$<$ http://www.conhecer.org.br/enciclop/2017a/sau/fatores\%20ambientais.pdf>. Acesso em: 02 out. 2020.

PNUD - PROGRAMA DAS NAÇÕES UNIDAS PARA O DESENVOLVIMENTO. Entenda o Atlas. Brasilia: Organização das Nações Unidas. Atlas do Desenvolvimento Humano no Brasil. Organização das Nações Unidas. 2020. Disponível em: $<$ https://www.br.undp.org/content/brazil/pt/home/idh0/atlas-do-desenvolvimentohumano/entenda-o-atlas.html>. Acesso em 03 jul. 2020.

ROCHA, R.C. Epidemiologia da dengue na cidade de Rio Branco- Acre, Brasil, no período de 2000 a 2007. [Tese]. São Paulo (SP): Universidade de São Paulo, 2011. Disponível em:

$<$ https://www.teses.usp.br/teses/disponiveis/6/6132/tde-28092011-135716/publico/RicardoRocha.pdf $>$. Acesso em: 29 set. 2020.

Secretaria de Estado de Saúde de Minas Gerais. Notícias.Boletim Epidemiológico de Monitoramento dos casos de Dengue, Chikungunya e Zika (08/01). 2020. Disponível em:<

https://www.saude.mg.gov.br/component/gmg/story/11977-boletim-epidemiologico-de-monitoramento-doscasos-de-dengue-chikungunya-e-zika-08-01>. Acesso em 23 abr. 2020. 
Secretaria de Estado de Saúde de Minas Gerais. Notícias.Boletim Epidemiológico de Monitoramento dos casos de Dengue, Chikungunya e Zika (14/01). 2020. Disponível

em:<https://www.saude.mg.gov.br/component/gmg/story/11986-boletim-epidemiologico-de-monitoramentodos-casos-de-dengue-chikungunya-e-zika-14-01>. Acesso em 23 abr. 2020.

SILVA, G.M.; ANDRADE, A.M.S.S. Avaliação do perfil epidemiológico da dengue no município de Paripiranga, Bahia, Brasil. Scientia Plena, [S.1], v.10, n.9, 2014. 